\title{
FOCAL MECHANISM IN CONNECTION WITH ENERGY STORAGE BEFORE CRACK FORMATION
}

\author{
R. DMOWSKA, K. RYBICKI and R. TEISSEYRE \\ Institute of Geophysics, Polish Academy of Sciences, Warsaw (Poland) \\ (Received January 8, 1972)
}

\section{ABSTRACT}

Dmowska, R., Rybicki, K. and Teisseyre, R., 1972. Focal mechanism in connection with energy storage before crack deformation. In: E.F. Savarensky and T. Rikitake (Editors), Forerunners of Strong Earthquakes. Tectonophysics, 14(3/4): 309-318.

The pattern of internal energy storage depends on distribution of the internal failures, fractures and local stress sources.

A simple model of energy storage along a tectonic plane is represented by an array of dislocations.

Stress concentration leads to crack formation of tensile, shear or transverse typc depending on the confining pressure and stress components prevailing at the tectonic plane.

The conditions of energy storage and conditions of crack formation vary with depth. Hence it is expected that the focal mechanism is also subject to transformation with depth.

\section{INTRODUCTION}

A complicated pattern of internal structure of the earth's crust and upper mantle, including failures, fractures and internal stress sources, causes remarkable local deviations in any regional stress field.

Dislocations and cracks represent phenomena described by material distortions and their own stress field. Total stress distribution is thus fairly complicated.

In this paper we assume that tectonic energy storage can be approximated by accumulation and concentration of dislocations. In a simple case the dislocations are grouped along a tectonic plane. A linear array of dislocations is formed when some factors prevent spreading of dislocations: intersections cutting the tectonic plane, inclusions or the layer boundaries can form such limits and in consequence dislocations are concentrated near the first locked dislocation. Array properties have been deduced by Eshelby et al. (1951).

The surface deformations due to the adopted model of tectonic energy storage, as well as the mechanism of earthquakes in the process of crack formations and its changes with depth are considered further in this paper.

\section{INCREMENTS OF SURFACE DEFORMATIONS}

Tectonic energy accumulation is revealed on the earth surface by a certain distribution 


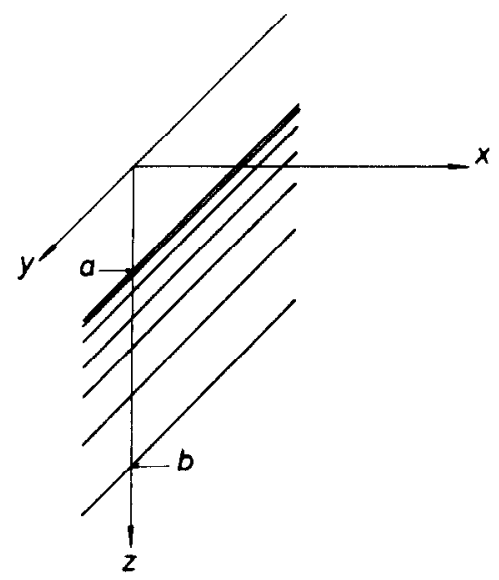

Fig. 1. Dislocation array with depth.

of deformations. Reversely, the pattern of an internal energy storage can be studied through surface deformations and in this way the problem becomes important for the earthquake prediction program.

With this aim in mind we will calculate the increments of surface deformations due to an increase of stress concentration for the case of a linear array of dislocations (Fig. 1; the earth surface is defined by $z=0$ ). The considered array comprises $n$ dislocations situated in the depth range $a \leqslant z \leqslant b$ (Fig. 1). The constant regional stress field ( $p_{x z}=\tau_{\mathrm{o}}$ in the case of edge dislocations, $p_{x y}=\tau_{0}^{\prime}$ in the case of screw dislocations) pushes dislocations towards the upper limit $z=a$ near the earth's surface. The screw dislocations are located here to transverse slip motion, while edge dislocations to normal/re;erse slips. Equilibrium conditions are assumed; the positions of dislocations are determined by a pushing force of regional stresses, repelling interaction between dislocations, influence of a free surface and the assumed condition that the first dislocation is locked at $z=a$. The solution is given after Head and Louat (1955) for a continuous dislocation distribution $f(z)$. The surface effect is included by the similar distribution of the image dislocations; this procedure is the exact one for a screw dislocation array and it can be used for the edge dislocations as the first approximation only (Head, 1953).

The solution is the following:

$$
f(z)=\frac{\tau_{\mathrm{o}}}{\pi A}\left(\frac{b^{2}-z^{2}}{z^{2}-a^{2}}\right)^{1 / 2}
$$

where $A=\mu \delta / 2 \pi$ for screw dislocations and $A=\mu \delta /\{2 \pi(1-\nu)\}$ for edge dislocations; $\mu=$ rigidity modulus, $\nu=$ Poisson ratio, $\delta=$ discontinuity displacement of dislocation. The condition joining continuous distribution with a discrete one reads: 

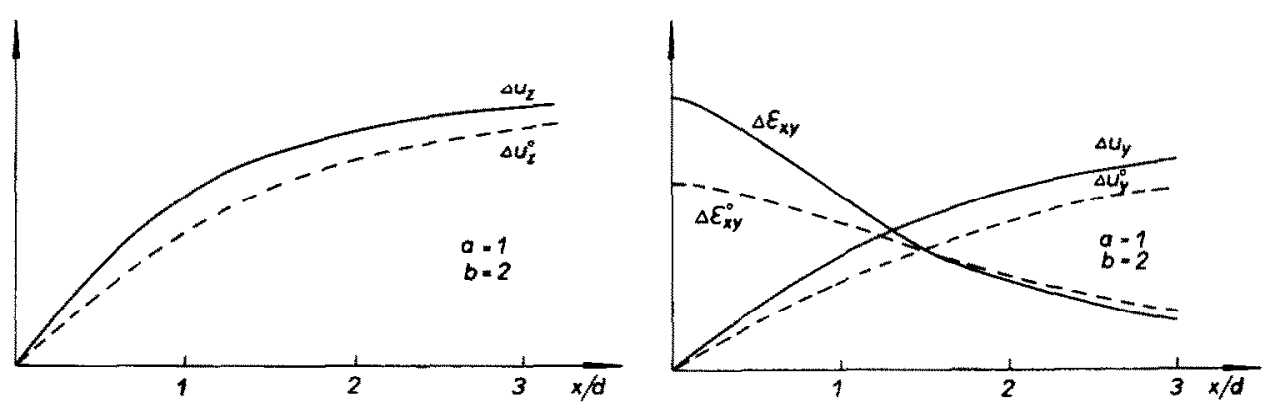

Fig. 2. Schematic graphical illustration of increments $\Delta U_{Z}, \Delta U_{Z}^{0}$ related to edge dislocations; $a=1, b=$ $2, d=1$.

Fig. 3. Schematic graphical illusuation of increments $\Delta U_{\mathrm{y}}, \Delta \epsilon_{\mathrm{zy}}, \Delta U_{\mathrm{y}}^{0}, \Delta \epsilon_{\mathrm{xy}}^{0}$ related to screw dislocations; $a=1, b=2, d=1$.

$$
n=\int_{a}^{b} f(z) \mathrm{d} z
$$

For the distribution of eq. 1 this condition is given by the elliptic integrals of firstly $K$ and secondly $E$ types:

$$
n=\frac{\tau_{\mathrm{o}} b}{\pi A}\left\{K\left[\left(1-\frac{a^{2}}{b^{2}}\right)^{1 / 2}\right]-E\left[\left(1-\frac{a^{2}}{b^{2}}\right)^{1 / 2}\right]\right)
$$

Now we can easily calculate the surface displacements and deformations using the known formulae for dislocation field with integration over the dislocation distribution within the limits of $a$ and $b$. However, we believe that more important for the prediction program is the study of the deformation increments which indicate the rate of energy accumulation.

The increments of surface displacements and deformations $(\Delta U, \Delta \epsilon)$ are here calculated on the assumption that the number of dislocations increases by $\Delta n$ at the lower point $z=b$. From eq. 3 we get:

$$
\Delta n=\frac{\partial n}{\partial b} \Delta b=\frac{\tau_{\mathrm{o}}}{\pi A} K\left[\left(1-\frac{a^{2}}{b^{2}}\right)^{1 / 2}\right] \Delta b
$$

In Fig. $2-4$ we present the examples of the increases of surface displacement $\Delta U_{y}$ and deformation $\Delta \epsilon_{\mathrm{xy}}$ (screw dislocations) and $\Delta U_{z}$ (edge dislocations). The presented curves are normalized to a unit length $d=b-a$ (two sets of values are assumed: $a=1, b=2$ and $a=$ $0.5, b=1.5)$. The units of $\Delta U$ and $\Delta \epsilon$ are not important in the present considerations and are taken arbitrarily. 


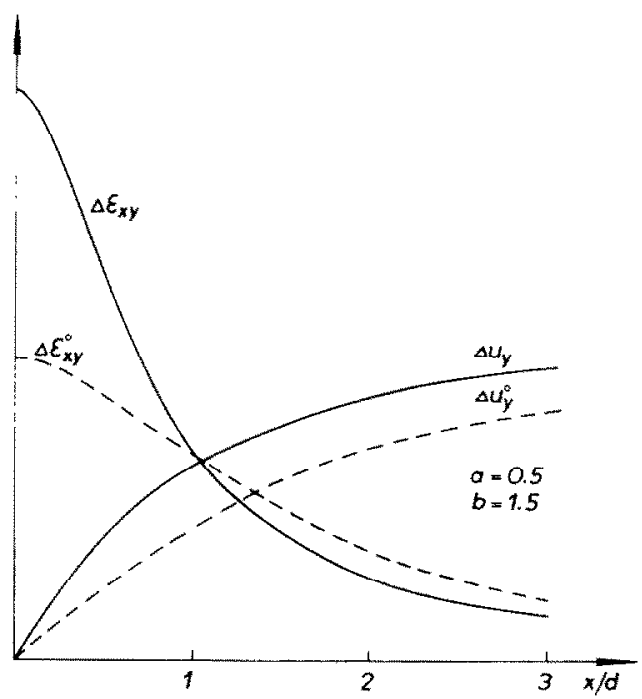

Fig. 4. Schematic graphical illustration of increments $\Delta U_{\mathrm{y}}, \Delta \epsilon_{\mathrm{xy}}, \Delta U_{\mathrm{y}}^{0}, \Delta \epsilon_{\mathrm{xy}}^{\mathrm{o}}$ related to screw dislocations; $a=0.5, b=1.5, d=1$.

The effect of stress concentration and pre-stress system on the increase of surface deformation can be seen when comparing it with surface deformation caused by the appearance of a single dislocation at $z=b$ with $\delta=\Delta n$ in a homogeneous half-space (no other dislocations present, i.e., an unstress system). To this aim the corresponding surface deformations $\Delta U^{\circ}, \Delta \epsilon^{\circ}$ due to a single dislocation at $z=b$ are simultaneously shown on Fig.2-4. The effect of stress concentration, as given by an array model, manifests here in greater values of $\Delta U, \Delta \epsilon$ in comparison with $\Delta U^{\circ}, \Delta \epsilon^{\mathrm{o}}$ on small distances and more rapid decrease of $\Delta U, \Delta \epsilon$ on larger distances.

\section{('RACK FORMATION AND) ENERGY RELEASE}

The dislocation model of stress concentration presented above is adequate to describe the possible occurrences of energy release. The process: dislocations $\rightarrow$ crack has been introduced as a process causing a seismic event in a former paper (Teisseyre, 1970). The total tectonic stresses at the appropriate level of concentration lead to fracture formation. In our representation the dislocations can form a crack while entering it. Eshelby et al. (1951) have shown that the stress concentration effect near the first locked dislocation is equal to the multiplication of the appropriate regional stress component $\tau_{\mathrm{o}}$ by a number $n$ of dislocations grouped in an array. The crack can thus be developed near a place of stress concentration at the active tectonic plane. The transformation dislocations $\rightarrow$ crack is accompanied by an energy release; the energy balance has been given by Stroh (1953, 1957). For different dislocation vectors $\delta_{i}$, where $i=$ index of dislocation, the corresponding formula has been generalized by Teisseyre (1964). Stroh in his papers considered edgedislocation array and tensile-crack formation only; Stroh (1954) showed that the elastic 
energy of the edge-dislocation array amounts to:

$$
E_{1}=\frac{\mu\left(\sum^{N} \delta_{i}\right)^{2}}{4 \pi(1-\nu)} \ln \frac{4 \pi e^{1 / 2}(1-\nu) \tau R}{\mu \sum^{N} \delta_{i}}
$$

where: $N=$ number of dislocations; $\tau_{0}=$ regional shear field assumed to act along a tectonic plane; $R=$ cut-off distance.

We assume that some of the dislocations have formed tensile crack; its full energy is given by Stroh (1957) and Teisseyre (1970):

$$
E_{2}=2 \gamma \lambda_{1} \ln \frac{4 R}{\lambda}+2 \gamma \lambda-\frac{\pi(1-\nu)}{8 \mu} \sigma^{2} \lambda^{2}-\frac{1}{2} \sigma \lambda \sin \alpha \sum^{n} \delta_{i}
$$

where: $\lambda_{1}=\{\mu / 8 \pi(1-\nu) \gamma\} \sum^{n} \delta_{i} ;$ and $\lambda_{2}=\{8 \mu \gamma / \pi(1-\nu)\}, n=$ number of dislocations which entered crack, $\gamma=$ surface crack energy, $\lambda=$ length of crack, and $\sigma=$ tensile field perpendicular to crack, i.e., $\sigma=\sigma_{1} \cos ^{2} \alpha+\sigma_{\|} \sin ^{2} \alpha+\tau_{0} \sin ^{2} \alpha ; \sigma_{\|}, \sigma_{1}=$ tensile regional stresses parallel and perpendicular to tectonic plane, respectively, $\alpha=$ angle between crack and tectonic plane. The energy release of the dislocations $\rightarrow$ crack process is according to eq. 5 and 6 :

$$
\Delta E=2 \gamma \lambda_{1} \ln \frac{e^{1 / 2} \tau_{0} \lambda \xi}{\left(\lambda_{1} \lambda_{2}\right)^{1 / 2}}-2 \gamma \lambda+\frac{\gamma}{\lambda_{2}} \sigma^{2} \lambda^{2}+4 \gamma \sin \alpha\left(\frac{\lambda_{1}}{\lambda_{2}}\right)^{1 / 2} \sigma \lambda
$$

where: $\xi=$ ratio $\sum^{n} \delta_{i} / \sum^{N} \delta_{i}$.

In seismological problems not only the tensile crack is important; we can consider three types of cracks depending on the type of dislocations and stresses prevailing near the tectonic plane. Edge dislocations form tensile or shear cracks depending on the regional stresses and the confining hydrostatic pressure. Screw dislocations can form a transverse crack (two dimensional case) related to strike-slip motion.

We can now derive for shear and transverse cracks formulae for energy release similar to eq. 7 for tensile cracks.

The contribution to crack energy in the field of external stresses can be deduced from the crack-field formulae given by Matczynski (1970). The simple integration $\frac{1}{2} \iint \Delta U_{i} p_{i k} \mathrm{~d} s_{k}$ over a crack surface gives a similar result for crack energy as in the case of the tensile crack (third term in eq.6), while the last term in eq. 6 related to volume increase appears only in the case of tensile cracking. Thus, the elastic crack energy is decreased by term $\pi(1-\nu) \tau^{2} \lambda^{2} / 8 \mu$ for shear and $\pi \tau^{\prime 2} \lambda^{2} / 8 \mu$ for a transverse crack, where $\tau$ and $\tau^{\prime}$ are the appropriate shear components related to crack surface.

The expression for shear and tensile crack energy can be now presented in one formula which takes into account the angle that exists between the crack and the tectonic surface: 


$$
E_{2}=2 \gamma \lambda_{1} \ln \frac{4 R}{\lambda}+2 \gamma \lambda-\frac{\pi(1-\nu)}{8 \mu}\left(\sigma^{2}+\tau^{2}\right) \lambda^{2}-\frac{1}{2} \sigma \lambda \sin \alpha \sum^{n} \delta_{i}
$$

where: $\sigma^{2}+\tau^{2}=\tau_{0}^{2}+\sigma_{1}^{2} \cos ^{2} \alpha+\sigma_{1}^{2} \sin ^{2} \alpha+\tau \sigma_{\|} \sin 2 \alpha+\tau \sigma_{1} \sin 2 \alpha$. Here stress components $\tau_{0}, \sigma_{1}, \sigma_{\|}$are related to a tectonic plane, but the same result can be got for any reference system: surface energy $\gamma$ comprises here shear and tensile components. For the energy balance in the process of formation of tensile and shear cracks we get:

$$
\Delta E=2 \gamma \lambda_{1} \ln \frac{c^{1 / 2} \tau_{0} \lambda \xi}{\left(\lambda_{1} \lambda_{2}\right)^{1 / 2}}-2 \gamma \lambda+\frac{\gamma}{\lambda_{2}}\left(\sigma^{2}+\tau^{2}\right) \lambda^{2}+4 \gamma \sin \alpha\left(\frac{\lambda_{1}}{\lambda_{2}}\right)^{1 / 2} \sigma \lambda
$$

The elastic energy of screw dislocation array is given by a similar formula to eq. 5 but the factor $(1-\nu)$ should be omitted; similarly for a transverse crack we get analogous formulae to eq. 8 and 9 . Thus we have for energy difference:

$$
\Delta E^{\prime}=2 \gamma \lambda_{1}^{\prime} \ln \frac{e^{1 / 2} \tau_{0}^{\prime} \lambda \xi}{\left(\lambda_{1}^{\prime} \lambda_{2}^{\prime}\right)^{1 / 2}}-2 \gamma \lambda+\frac{\gamma}{\lambda_{2}^{\prime}} \tau^{\prime 2} \lambda^{2}
$$

where: $\lambda_{1}^{\prime}=\frac{\mu}{8 \pi \gamma} \sum^{n} \delta_{i} ; \lambda_{2}^{\prime}=8 \mu \gamma / \pi ;$ and $\tau_{\mathrm{o}}^{\prime}=$ tegional shear field (it is assumed he re that $\tau_{\mathrm{o}}^{\prime}$ acts along a tectonic plane) $\tau^{\prime}=$ shear stress related to a crack surface $\tau^{\prime}=\tau_{0}^{\prime} \sin \alpha$.

In the equations for energy balance for tensile and shear (eq.9) and transverse crack (eq.10) there appears a surface energy $\gamma$. This energy plays the role of material constant and it should be noted that $\gamma$ has different values for different types of static cracks.

Considering homogeneous space we find that the direction of crack development is related to its character and depends on stress conditions; a transverse crack will develop either in the same tectonic direction or deviate according to stress and structural conditions (Fig.5), a shear crack will lie rather in the tectonic direction, while a tensile crack will grow rather in a perpendicular plane (Fig.6). Considering for example an array consisting of edge dislocations subject to a constant shear stress $\tau_{0}$ related to the direction of the tectonic plane, we find that the most probable angle at which a tensile crack will develop is given by the maximum of tensile stresses perpendicular to the crack; the problem has been solved by Stroh (1954); a tensile crack is expected to develop at an angle of $70.5^{\circ}$ to the tectonic plane.

\section{DIRECTION OF CRACK DEVELOPMENT AND ITS CHANGES WITH DEPTH}

We propose now to study the part of tensile and shear components of a crack formed in an arbitrary stress field. To this aim we will determine the direction of crack development.

The proposed method concerns a mechanism of crack formation of tensile and shear type, for an elastic half-space with an arbitrary stress pattern. We denote the angle $\alpha$ 

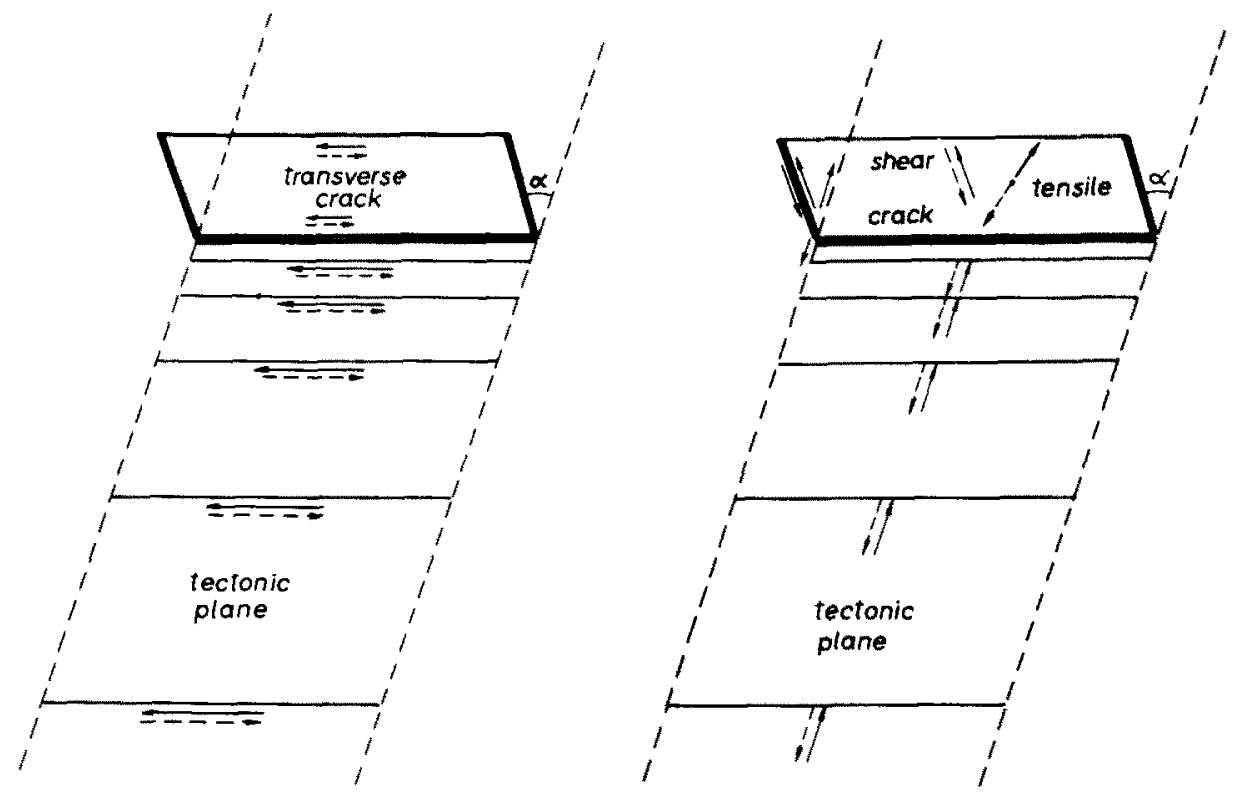

Fig. 5. Screw dislocation array and transformation to transverse crack.

Fig. 6. Edge dislocation array and transformation to shear and tensile crack.

between surface and $z$-axis of the Cartesian coordinate system as described in Fig.7. We shall consider here the stress $\sigma$ normal to the crack surface and the shear stress $\tau$ along it, when both are described (for a case of plane stress) as follows:

$$
\begin{aligned}
& \sigma=\sigma_{\mathrm{xx}} \cos ^{2} \alpha+\sigma_{\mathrm{zz}} \sin ^{2} \alpha+\sigma_{\mathrm{xz}} \sin 2 \alpha \\
& \tau=\frac{1}{2}\left(\sigma_{\mathrm{zz}}-\sigma_{\mathrm{xx}}\right) \sin 2 \alpha+\sigma_{\mathrm{xz}} \cos 2 \alpha
\end{aligned}
$$

The crack surface energy $\gamma$ will now be considered separately as tensile $\gamma_{1}$, and shear, $\gamma_{2}$, components. The relationship between crack surface energies $\gamma_{1}$ and $\gamma_{2}$ and critical values of stress components $\sigma$ and $\tau$ are of the form:

$$
\begin{array}{ll}
2 \gamma_{1}=\frac{\pi(1-\nu)}{2 \mu} k_{1}^{2} ; & k_{1}=\frac{\sigma(2 \lambda)^{1 / 2}}{\pi} \\
2 \gamma_{2}=\frac{\pi(1-\nu)}{2 \mu} k_{2}^{2} ; & k_{2}=\frac{2 \tau \lambda^{1 / 2}}{\pi(2-\nu)}
\end{array}
$$

Here $\gamma_{1}$ and $\gamma_{2}$ are considered as material constants not dependent on the velocity of 
crack propagation (static case), $k_{1}$ and $k_{2}$ are stress-intensity factors, and $\sigma$ and $\tau$ describe critical normal and critical shear stress, which could cause the crack propagation.

Since critical values of stress field $\sigma$ and $\tau$ depend on depth $z$, measured from the surface of the half-space and on the value of the angle $\alpha$, there should be a connection between these quantities and energy $e$ for a unit of crack surface (equal here to the sum of surface energies for pure tensile and pure shear modes, $2 e=2 \gamma_{1}+2 \gamma_{2}$ ):

$$
e(\sigma, \tau) \rightarrow e(z, \alpha)
$$

We introduce here the condition stating that such a crack develops first which has a maximum value of surface energy:

$$
\left(\frac{\partial e(z, \alpha)}{\partial \alpha}\right)_{z}=0
$$

In this way such $\alpha_{0}=\alpha_{0}(z)$ can be found, which corresponds to the minimum difference between internal energies before and after formation of a new crack surface. $\alpha_{0}(z)$ describes the most probable angle of the new crack; both tensile and shear crack formation mechanisms being taken into account.

As an example of this method two different stress fields are considered.

In the first case we considered the elastic layer of $1000 \mathrm{~km}$ thickness and the stress distribution, which is the sum of hydrostatic pressure, calculated for the upper $100 \mathrm{~km}$ of the earth by Bullen and Haddon (1970), and thermal stress field given by Lubimova (1963) (Fig.8). The $\mu$ and $\nu$ depth dependences are after Bullen (1953).
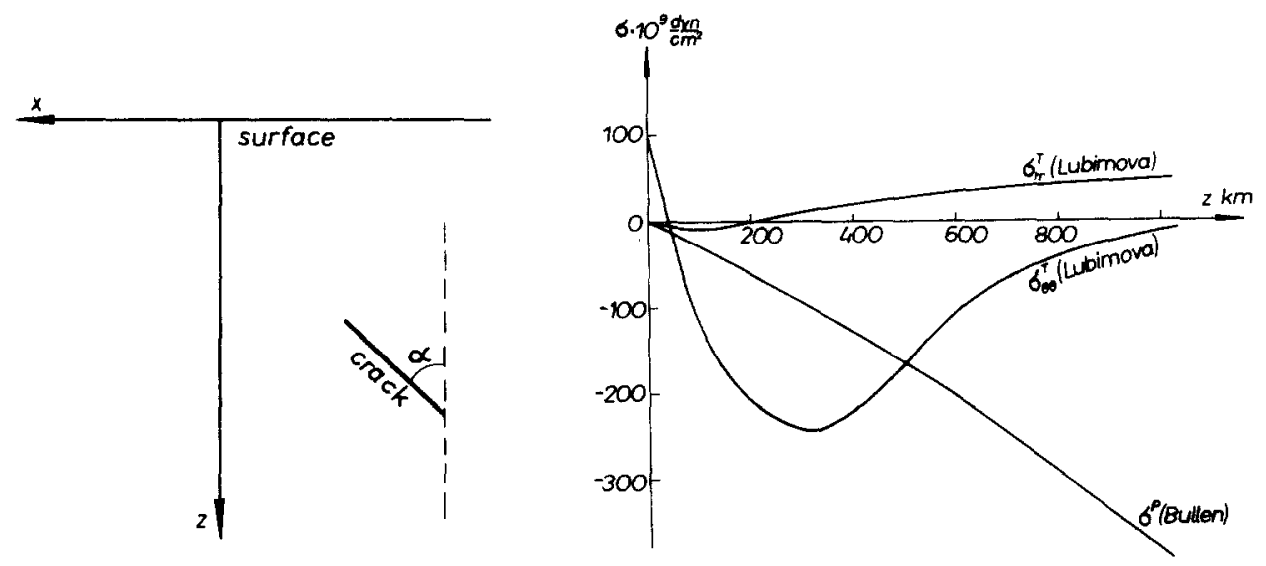

Fig. 7. Crack in the reference system $x z$.

Fig. 8. Stress distribution in the earth's interior. 


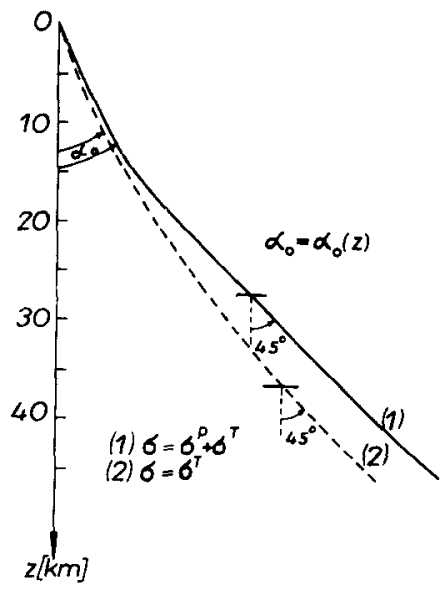

Fig. 9. Inclination angle of cracks as function of depth in the cases determined by thermal stresses and hydrostatic pressure and by thermal stresses only.

Using the proposed method special attention must be paid to the region in which tensile mechanism of crack creation is possible, i.e., $\sigma(z, \alpha)>0$. For the stress field under discussion this region is as follows:

$$
\begin{array}{ll}
0<z \leqslant 35 \mathrm{~km} & \text { for } \alpha_{0}=0 \\
0<z \leqslant 27.5 \mathrm{~km} & \text { for } \alpha_{\mathrm{o}}=\pi / 4
\end{array}
$$

So for this region we assume that both tensile and shear mechanisms could act simultaneously. For the deeper part of the layer only shear cracks were thought possible.

The resulting $\alpha_{\mathrm{o}}(z)$ dependence is shown by the solid line in Fig.9.

In the second case there are considered the thermal stresses only, the same as in the first case. The region, in which both crack creation mechanisms could be possible is as follows:

$$
\begin{array}{ll}
0<z \leqslant 40 \mathrm{~km} & \text { for } \alpha_{0}=0 \\
0<z \leqslant 36.5 \mathrm{~km} & \text { for } \alpha_{0}=\pi / 4
\end{array}
$$

The resulting $\alpha_{\mathrm{o}}(z)$ dependence is shown by the dotted line in Fig.9.

The above proposed method can be applied to an arbitrary stress distribution, in particular to a local one disturbed by stress concentration due to dislocation array.

The same method based on condition $(\partial e / \partial \alpha)_{z}=0$ can be applied to determine a direction of transverse crack development in relation to the corresponding shear stress component $\tau_{\mathrm{o}}^{\prime}$. 
The crack directions, as determined above, have statistical meaning; these directions are related to the most probable cases.

\section{REFERENCES}

Bullen, K.E., 1953. An Introduction to the Theory of Seismology. Cambridge University Press, London and New York, 2nd. ed. 296 pp.

Bullen, K.E. and Haddon, R.A.W., 1970. Evidence from seismology and related sources on the earth's present internal structure. Phys. Earth Planet. Inter., 2: 342-349.

Eshelby, I.D., Frank, F.C. and Nabarro, F.R.N., 1951. The equilibrium of linear arrays of dislocation. Philos. Mag., 42: 351-364.

Head, A.K., 1953. The interaction of dislocations and houndaries. Philos. Mag., 44: 92-94.

Head, A.K. and Louat, N., 1955. The distribution of dislocations in linear arrays. Austral. J. Phys., 8: $1-7$.

Lubimova, H.A., 1963. Distribution of thermoelastic stresses within the earth and their rate of accumulation. Akad. Nauk S.S.R. Izv., Ser. Geofiz, 3: 385-390 (in Russian).

Matczyniski, M., 1970. The static problem of a crack in an elastic medium. Arch, Mech. Stos./Arch. Mecan. Appl), 22: 439-478 (article in English).

Stroh, A.N., 1953. A theoretical calculation of the stored energy in a work-hardened material. Proc. R. Soc. London, ser. A., 218: 391-400.

Stroh, A.N., 1954. The formation of cracks as a result of plastic flow. Proc. R. Soc. London, ser. A, 223: $404-414$.

Stroh, A.N., 1957. A theory of the fracture of metals. Adv. Phys., 6:418-465.

Teisseyre, R., 1964. Dislocation systems and their interaction in shock sequence. Acta Geophys. Polon., 12 (1): 23-36 (in English).

Teisseyre, R., 1970. Crack formation and energy release caused by the concentration of dislocations along fault planes. Tectonophysics., 9: 547-557. 